\title{
Herramienta para la enseñanza de la geometría utilizando Tics, dirigido a los estudiantes del segundo año de bachillerato
}

\begin{abstract}
Tool for teaching geometry using Tics, aimed at students in the second year of high school
\end{abstract}

Jorge Cachuput Gusñay. ${ }^{1}$, Juan Manuel Martínez Nogales. ${ }^{2}$, Jaime Rodrigo Guilcapi Mosquera. ${ }^{3} \&$ Jorge Rigoberto López Ortega. ${ }^{4}$

\begin{abstract}
.
Development of a didactic guideline as tool for Geometry teaching by using TICS, directed to the students of Second Bachelorship Year of the Education Unit "Pedro Vicente Maldonado", in function to the educational problem presented, a questionnaire was formulated by applying the TICS as a didactic resource in the teaching of Geometry, with this tool was applied the survey to the students and teachers, to make the didactic guideline by using the educational software GeoGebra, therefore the importance to make meaningful designs based on the scholar level and with contents related to the planning and programs of Geometry updated in the educational sector. According to the work experience, a didactic guideline was designed for the use of educational software, specifically for Geometry. It is concluded that in the investigation made the $72 \%$ of surveyed students consider that by using TICS they would improve their academic performance. It is recommended that training the

\footnotetext{
${ }^{1}$ Escuela Superior Politécnica de Chimborazo, Facultad de Recursos Naturales. Riobamba, Ecuador. jorge.cachuput@espoch.edu.ec

${ }^{2}$ Escuela Superior Politécnica de Chimborazo, Facultad de Recursos Naturales. Riobamba, Ecuador. jumartinez@espoch.edu.ec

${ }^{3}$ Universidad Técnica de Amato, Facultad de Ciencias de la Educación, Carrera de Cultura Física. Ambato, Ecuador.jr.guilcapi@uta.edu.ec

4 Escuela Superior Politécnica de Chimborazo, Facultad de Recursos Naturales. Riobamba, Ecuador. jorge.lopezo@espoch.edu.ec
} 
teacher in the area and promote in the institution that this would be able to be applied in the teaching-learning process.

Keywords: Technologies of information and communication tics, informatic resource, teaching learning, geometry mathematics, software GeoGebra

\section{Resumen}

Desarrollo de una guía didáctica como herramienta para la enseñanza de la geometría utilizando tics, dirigido a los estudiantes del segundo año bachillerato de la unidad educativa "Pedro Vicente Maldonado" de la ciudad de Riobamba, en función a la problemática de la educación, se formuló un cuestionario de preguntas aplicando las TICS como recurso didáctico en la enseñanza de la geometría, con esta herramienta se aplicó la encuesta a los estudiantes y docentes, para realizar la guía didáctica utilizando el software educativo Geogebra, de ahí la importancia de realizar diseños llamativos acordes al grado escolar y con contenidos apegados a los planes y programas de geometría vigentes en el sector educativo. De acuerdo a la experiencia de trabajo, se diseñó una guía didáctica para el uso del software educativo específicamente para la geometría. Se concluye la investigación realizada que el $72 \%$ de estudiantes encuestados consideran, usando las TICS mejoraría el aprendizaje de la geometría y su rendimiento académico. Se recomienda capacitar a los docentes del área y promover la propuesta en la institución para que pueda ser aplicado en el proceso enseñanza aprendizaje.

Palabras Claves: Tecnologías, tics, recurso informático, enseñanza aprendizaje, geometría matemática, software GeoGebra.

\section{Introducción.}

Las TICS (Tecnologías de la Información y Comunicación), son instrumentos y materiales que facilitan el aprendizaje, el desarrollo de habilidades y diferentes formas de aprendizaje, el desarrollo de habilidades y diferentes formas de aprender, estilos y ritmos de los aprendices.

Las tecnologías de la información y la comunicación (TIC) pueden contribuir al acceso universal a la educación, la igualdad en la instrucción, el ejercicio de la enseñanza y el aprendizaje de calidad y el desarrollo profesional de los docentes, así como a la gestión dirección y administración más eficientes del sistema educativo.

Nos permite:

- Educación sincrónica y asincrónica.

- Las TICS como herramientas de compilación, análisis y procesamiento de información.

- Beneficia el trabajo cooperativo.

- Uso eficiente y constante de los recursos de sistematización e informática. 
- Trae el mundo al aula de clases y lleva el aula al ámbito global.

- Nuevos esquemas de gestión de conocimiento.

- Soporte hacia implementar nuevos métodos de enseñanza-aprendizaje e investigación.

La importancia de la presente investigación está centrada en el estudio de la aplicación de las tics como recurso didáctico en la enseñanza de la geometría a fin de mejorar el rendimiento académico dirigido a los estudiantes del segundo año de bachillerato de la Unidad Educativa "Pedro Vicente Maldonado", con la utilización del Geogebra en la parte de geometría con la finalidad de buscar estrategias innovadoras para los estudiantes que permitan desarrollar sus capacidades para que puedan comprender, asociar, analizar e interpretar los conocimientos adquiridos.

Para ello se determina la situación problemática actual en cuanto a la utilización de las tics con el objetivo de aprovechar el potencial del estudiante dentro del proceso de enseñanzaaprendizaje como también de los docentes para impartir la clase en el área de matemática, ya que las estrategias que utilicen deben ser las más adecuadas para transmitir los contenidos a los estudiantes.

El objetivo fundamental de este estudio es analizar el proceso integral del estudiante en el desarrollo de habilidades y destrezas básicas para facilitar la interpretación del medio que lo rodea siendo condición necesaria para la convivencia social tanto para el docente como para el estudiante; donde el docente desarrolla el autoestima de los educandos en la aplicación de estrategias de enseñanza de la matemática.

El aprendizaje obedece de la relación sujeto mundo, por tanto el hombre desde su nacimiento comienza a apropiarse de esa realidad en un continuo proceso de aprendizaje, la retención que se produce a través del conocimiento, pero si nos damos cuenta desde que nace el hombre necesita de la guía, de la dirección de otro que interprete sus necesidades y en correspondencia actúe para poder satisfacerla.

Según Carlos Álvarez de Zayas, opina en su libro Pedagogía como Ciencia, que el aprendizaje es la actividad que desarrolla el estudiante para aprender, para asimilar la materia de estudio, por su parte la instrucción es referida a la actividad que ejecuta el profesor, sin embargo, en el proceso docente educativo tradicional el estudiante se convierte en objeto del proceso por lo que no se manifiesta lo más importante que este se inserte en el proceso como sujeto de su propio aprendizaje.

No hay enseñanza sin aprendizaje y viceversa; ambos se ejecutan en un ambiente activo. Sin actividad no hay enseñanza, ni aprendizaje. La actividad del proceso educativo se produce por los sujetos: estudiantes y maestros, con toda su carga de subjetividad, encerrando los 
afectos; el método es el mecanismo didáctico que recoge la acción intelectual de los sujetos, pero también la vivencial y afectiva; al menos en una didáctica constructiva, significativa, crítica.

Una concepción didáctica científico-crítica. Estamos hablando de una enseñanza activa y un aprendizaje activo. (Castellanos, 2005)

Figura 1 Concepción dialéctica integral

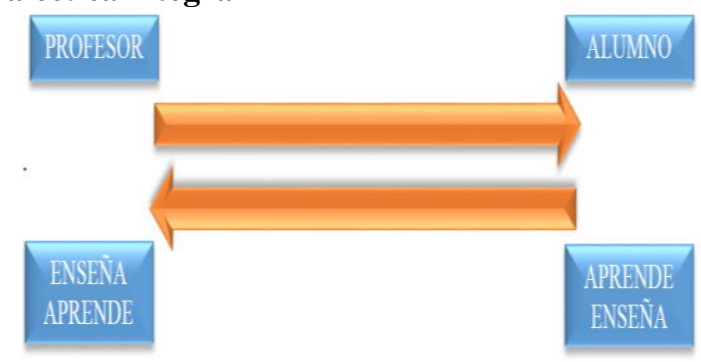

Fuente: Concepción de dialéctica integral (Guzmán, 1991)

Un proceso dialécticamente integral, que da respuesta a las exigencias del aprendizaje de conocimientos, del desarrollo intelectual y físico del estudiante y a la formación de sentimientos cualidades y valores, todo lo cual da cumplimiento en sentido general y en particular a los objetivos propuesto para cada nivel y del modelo del profesional. (Guzmán, 1991)

La UNESCO aplica una estrategia amplia e integradora en lo tocante a la promoción de las TIC en la educación. El acceso, la integración y la calidad figuran entre los principales problemas que las TIC pueden abordar. El dispositivo intersectorial de la UNESCO para el aprendizaje potenciado por las TIC aborda estos temas mediante la labor conjunta de sus tres sectores: Comunicación e Información, Educación y Ciencias.

La asociación de la información digital con la computación ha enriquecido la propia esencia de la información y ha tolerado el surgimiento de la llamada información multimedia en la cual el texto consigue ir acompañando de imágenes, sonido y video.

La formación es un mecanismo esencial en el proceso de incorporar las nuevas tecnologías a las acciones cotidianas. A través de esta nueva forma de enseñanza el estudiante y el docente pueden administra sus tiempo, hablamos de una educación asincrónica.

Los beneficios más claros que los medios de comunicación contribuyen a la sociedad se encuentran el acceso a la cultura y a la educación, donde los avances tecnológicos y los beneficios que permite la era de la comunicación lanzan un balance y unas previsiones asombrosamente positivas. Por esto, es provechoso una adecuada educación en el uso de estos poderosos medios.

En el empeño por reforzar el aprendizaje, las TIC se usan cada vez más para personalizarlo, diferenciar la instrucción, fomentar el aprendizaje en contextos extraescolares, compartir 
recursos, colaborar, dinamizar las evaluaciones y cambiar el uso de las aulas. Pero la repercusión de estas innovaciones todavía está por evaluarse, reconocerse y ampliarse.

El Premio UNESCO-Rey Hamad bin Isa Al Khalifa para la Utilización de las Tecnologías de la Información y la Comunicación en la Educación , creado en 2005 bajo los auspicios del Reino de Bahrein, recompensa a particulares, instituciones y organizaciones no gubernamentales que realizan proyectos y actividades en los que se hayan aplicado creativamente prácticas idóneas en materia de tecnologías de la información y la comunicación (TIC) con miras a fomentar el aprendizaje, la enseñanza y los resultados educativos en general.

Este es el único premio de la UNESCO en el ámbito de la aplicación de las TIC en la educación y busca reconocer a las organizaciones y los particulares que las utilizan como instrumento pedagógico y, a la vez, tratan de aumentar la eficacia del aprendizaje. Al tiempo que reconoce la importancia de las innovaciones didácticas que las TIC propician o que se apoyan en ellas, es fundamental que dichas innovaciones garanticen la seguridad de los niños y promuevan los valores y las actitudes pertinentes para la construcción de sociedades sostenibles y pacíficas.

Esta tendencia ha tenido un gran predominio en EEUU donde surge, así como en otros países. En América Latina, ha sido divulgada ampliamente debido a la influencia que los sistemas norteamericanos de enseñanza tienen en nuestro país. Este predominio opera en nuestro país en tres campos: en la computación, la información y las comunicaciones.

Por lo tanto, la presente propuesta permitirá analizar el proceso integral del estudiante en el desarrollo de habilidades y destrezas básicas para facilitar la interpretación del medio que lo rodea siendo condición necesaria para la convivencia social tanto para el docente como para el estudiante; donde el docente desarrolla el autoestima de los educandos en la aplicación de estrategias de enseñanza de la matemática.

Espero que esta investigación sea una pequeña contribución para mejorar la calidad de enseñanza en la algebra y que sirva de recurso para desarrollar el pensamiento de los estudiantes como estímulo para potenciar sus capacidades.

\section{Marco teórico de referencia}

Las directrices conductuales (asociacionistas) sobre el aprendizaje matemático reflexionan que aprender es cambiar conductas, insisten en destrezas de cálculo y dividen estas destrezas en pequeños pasos para que, mediante el aprendizaje de destrezas simples, se llegue a aprender sucesiones de destrezas más complejas.

Las investigaciones sobre el aprendizaje matemático en el asociacionismo son muy nutridas, ya que las matemáticas brindan un campo propenso a estudiar el éxito o fracaso en la tarea. Gran segmento de estas investigaciones tienen como fin determinar la dificultad de una tarea 
matemática, para lo cual se observan las edades a las que los alumnos conseguían una mayoría de éxitos. También se ha investigado sobre cuál es la dominante secuencia de aprendizaje, es decir, qué actividades hay que realizar para aprender, y en qué orden hay que desarrollarlas.

Una de las teorías asociacionistas más significativas en correspondencia con el aprendizaje de las matemáticas es la de Gagné. Este autor trata de establecer jerarquías de aprendizaje, trata de organizar las lecciones de acuerdo con la complejidad de las tareas, para conseguir un mayor número de éxitos. Para ello planifica la lección descomponiendo la conducta que hay que aprender en partes más simples, y las organiza jerárquicamente en una secuencia de instrucción. Gagné llama secuencia de instrucción a una cadena de capacidades o destrezas ligadas a la capacidad superior que se quiere lograr. Esta cadena empieza destacando las destrezas que tienen que estar aprendidas para poder abordar los aprendizajes perseguidos (prerrequisitos), y continúa después delimitando los conceptos y, por último, las destrezas que se van a ejercitar. (Schwartz, 1995).

La geometría es una parte de la matemática encarga de estudiar las propiedades y las medidas de una figura en un plano o en un espacio

Si bien el estudio de la matemática es vital en la enseñanza primaria y secundaria, es una ciencia deductiva pura que se apoya en la experiencia. A raíz de mi investigación puedo inferir que los antecedentes han introducido conductas negativas en los estudiantes a nivel de la concepción del área de matemáticas como el área más compleja y que por lo tanto requiere de mentes inteligentes para lograr comprender y asimilar sus contenidos; lo anterior hace que el estudiante demuestre apatía por el área, manifieste rechazo, pereza e incluso indiferencia y desconocimiento de la aplicabilidad del área en sus vidas.

El estudio se basada en la formación de talento humano y en la generación de conocimiento e innovación científica utilizando nuevas tecnologías de información y comunicaciones, por ende con buenas prácticas y nuevas herramientas de producción, con lo cual se pone énfasis en el bio-conocimiento y en su aplicación a la producción de bienes y servicios ecológicamente sustentables. Estos procesos se orientan en función de la satisfacción de las necesidades del país.

Además la educación es un derecho de las personas a lo largo de su vida y un deber ineludible e inexcusable del estado ecuatoriano, Constituyendo un área prioritaria de la política pública y de la inversión estatal, garantía de la igualdad e inclusión social y condición indispensable para el Buen Vivir (Sumak Kausay). Las personas, las familias y la sociedad tienen el derecho y la responsabilidad de participar en el proceso educativo" (art. 26).

Cabe indicar que la educación no es un fin en sí mismo, es un proceso continuo y de interés público que integra todos los niveles de formación. El Sistema Nacional de Educación que comprende la educación inicial, básica y el bachillerato (art. 343) y el Sistema de Educación 
Superior (art. 350) están llamados a consolidar las capacidades y oportunidades de la población y a formar académica y profesionalmente a las personas bajo una visión científica y humanista, que incluye los saberes y las culturas de nuestro pueblo. A estos dos sistemas se suma la formación continua y la capacitación profesional del individuo.

En nuestro país, desde 1996 hasta el año 2007, se han aplicado, en cuatro ocasiones, las pruebas aprendo a los estudiantes de los años: tercero, séptimo y décimo de Educación Básica del sistema escolarizado, en las áreas de Matemática y Lenguaje y Comunicación, estas pruebas se aplicaron de manera muestral y estuvieron fundamentadas en la Teoría Clásica de los Test. En este contexto, el Ministerio de Educación oficializó a partir del 4 de junio de 2008, la implementación de las pruebas SER ECUADOR, para la evaluación del desempeño de los estudiantes, con la adopción de una nueva metodología: la teoría de Respuesta al Ítem (TRI) 1 en el desarrollo de las pruebas de logros académicos y los cuestionarios de factores asociados.

Por primera vez se aplicó estas pruebas en el año 2008, de manera censal, a estudiantes de establecimientos educativos fiscales, fisco misionales, municipales y particulares, a los años: cuarto, séptimo y decimo de Educación General Básica, y tercero de bachillerato, en las áreas de Matemática y Lenguaje y Comunicación, y se incluyó con las áreas de Estudios Sociales y Ciencias Naturales, de manera muestral, en los años: séptimo y decimo de educación básica.

Con estos antecedentes es necesario realizar el trabajo de elaboración una guía didáctica como herramienta de enseñanza de la geometría utilizando software interactivo Geogebra, para los estudiantes de segundo año bachillerato Unidad Educativa "Pedro Vicente Maldonado" de la ciudad de Riobamba.

Es preciso centrar los esfuerzos en garantizar el derecho a la educación a todos, en condiciones de calidad y equidad, ubicando en el centro al ser humano y al territorio. Para fortalecer el rol del conocimiento promoviendo a la investigación científica y tecnológica responsable con la sociedad y con la naturaleza.

Por consiguiente la guía didáctica será un recurso atractivo y fácil de utilizar, para los estudiantes de la Unidad Educativa "Pedro Vicente Maldonado", es una herramienta adecuada en este proceso de que hacer educativo, para ello se utiliza Geogebra como software interactivo en el proceso de enseñanza aprendizaje de la geometría, aplicando en forma individualizada y en equipo, respetando el ecosistema, la interculturalidad y la plurinacionalidad.

Los beneficiados del presente trabajo de investigación serán: los estudiantes a partir de la motivación y aplicación del tic's, que ayudará al mejor desenvolvimiento en el razonamiento matemático del estudiante. Los docentes de matemática por cuanto facilitará la enseñanza a 
los estudiantes a su cargo. Los padres de familia porque notarán cambios en sus hijos mediante sus rendimiento académico en la matemática.

El presente trabajo de investigación será de utilidad para mejorar el nivel académico de los estudiantes de la Unidad Educativa "Pedro Vicente Maldonado", ya que la propuesta en marcha servirá de apoyo para las autoridades y profesores de otras Instituciones con similares características.

La misión que se tiene como impulsadores del aprendizaje es construir el conocimiento, que le permita al estudiante desarrollar su imaginación e percepción por medio del razonamiento; lo que implica que los estudiantes aprendan a observar, comparar, clasificar, reunir y organizar datos, resumir, buscar supuestos, formular hipótesis, aplicar principios a nuevas situaciones, formular críticas, toma de decisiones, crear, interpretar, informar, criticar y evaluar, explicar su realidad social y física y esto se puede dar de mejor manera con la ayuda de la geometría.

En las secciones anteriores hemos considerado a la geometría principalmente como una teoría matemática y hemos analizado algunos aspectos de su enseñanza. Dado que el aprendizaje es incuestionablemente el otro polo esencial de cualquier proyecto educativo, es apropiado poner la debida atención a las principales variables que intervienen en un proceso coherente de enseñanza - aprendizaje.

La dimensión cognitiva, los procesos con los cuales, partiendo de la realidad, se conduce gradualmente hacia una percepción más refinada del espacio.

La dimensión epistemológica, la habilidad para explorar el inter juego entre la realidad y la teoría a través del modelado (hacer previsiones, evaluar sus efectos, reconsiderar selecciones). Es así que la axiomatización permite liberarse de la realidad; de esta manera puede ser vista como un recurso que facilita futuras conceptualizaciones.

La dimensión didáctica, la relación entre la enseñanza y el aprendizaje. En esta dimensión se encuentran muchos aspectos que merecen consideración. Como un ejemplo, listamos tres de ellos:

1) Hacer que interactúen varios campos (tanto al interior de la matemática como entre las matemáticas y otras ciencias).

2) Asegurar que los puntos de vista de los profesores y los estudiantes sean consistentes en un estudio dado. Por ejemplo, tener en cuenta que distintas escalas de distancia pueden involucrar diferentes concepciones y procesos adoptados por los estudiantes aun cuando la situación matemática sea la misma: En un "espacio de objetos pequeños", la percepción visual puede ayudar para hacer conjeturas y para identificar propiedades 
geométricas; cuando se está tratando con el espacio donde usualmente nos movemos (por ejemplo, el salón de clases) todavía resulta fácil obtener información local, pero puede dificultarse lograr una visión global; en un "espacio a gran escala" (como es el caso de la geografía o de la astronomía) las representaciones simbólicas son necesarias a fin de analizar sus propiedades.

3) Dar la debida consideración a la influencia de las herramientas disponibles en situaciones de enseñanza y de aprendizaje (desde la regla y compás tanto como otros materiales concretos, hasta calculadoras graficadoras, computadoras y software específico)

\section{Fundamentación filosófica}

El presente trabajo de investigación se basará en el Paradigma crítico-propositivo; el cual consiente en optimizar y mejorar la enseñanza aprendizaje de la matemática y el rendimiento académicos con la aplicación de las TICS en la geometría. Por lo tanto, su fundamentación es ontológica, epistemológica, axiológica y metodológica.

El paradigma se fundamenta en el hecho de que la vida social es dialéctica, no obligatoriamente debemos obtener un resultado científico; el método propuesto tiene como fin crear alternativas en la aplicación de un guía de geometría con las tics, utilizando el software Geogebra como herramienta de trabajo para perfeccionar la enseñanza aprendizaje de la matemática, partiendo de su comprensión y conocimiento, siguiendo un procedimiento metódico y sistemático, estableciendo una estrategia de gestión definida y con un enfoque investigativo para transformar su realidad social.

\section{Fundamentación ontológica}

Las unidades educativas deben formar a los estudiantes para que se conviertan en ciudadanos bien informados y profundamente motivados, provistos de un sentido crítico y capaz de analizar los problemas de la sociedad, buscar soluciones para los que se planteen a la sociedad, aplicar éstas y asumir responsabilidades sociales.

Se pretende desarrollar en el estudiante el aprendizaje autónomo y significativo, partiendo del interés y el deseo por aprender que tienen cada uno de los estudiantes, tomando como principio la motivación intrínseca y extrínseca de cada uno de ellos, seguido de la comprensión de los saberes, que implica la reflexión y el análisis de los mismos, al trabajar sobre la información, estudiando, analizando y aportando soluciones creativas, que generen una participación activa y en derivación aplicarlos a cada uno de los ámbitos de su vida, como son: personal, familiar, social, cultural, intelectual y moral de cada uno de los estudiantes. (Gimeno, 1992.)

Los estudiantes de la Unidad Educativa "Pedro Vicente Maldonado", enfrentan problemas desde la primaria, no tienen bases sólidas debido a la heterogeneidad de los grupos que ingresan a dicha entidad, también traen consigo otro tipo de problemas como son, la desorganización familiar, padres o madres que han viajado al exterior, no tienen control en 
su hogar por cuanto los padres trabajan, usan mal su tiempo libre en grupos inapropiados , pandillas, etc., son influenciables del medio; por lo que se observa mucha desmotivación.

La presente investigación está orientada a determinar el nivel de razonamiento con la aplicación de problemas de Matemática - geometría mediante el uso de las Tics lo que hará más fácil la comprensión y aprendizaje de la matemática, cosa que no se hace generalmente.

\section{Fundamentación epistemológica}

La misión que se tiene como impulsadores del aprendizaje es construir el conocimiento, que le permita al estudiante desarrollar su imaginación e percepción por medio del razonamiento; lo que implica que los estudiantes aprendan a observar, comparar, clasificar, reunir y organizar datos, resumir, buscar supuestos, formular hipótesis, aplicar principios a nuevas situaciones, formular críticas, toma de decisiones, crear, interpretar, informar, criticar y evaluar, explicar su realidad social y física y esto se puede dar de mejor manera con la ayuda de la geometría.

\section{Fundamentación axiológica}

Los maestros de matemática y geometría tiene la obligación de a más de transferir el conocimiento, desarrollar el pensamiento del estudiante, por lo tanto se pretende motivar al estudiante a aplicar las tics en la geometría para obtener un sobresaliente razonamiento, a más de verlo reflejado en un aprendizaje significativo y una educación de calidad, valorando en el estudiante el esfuerzo y dejándolo actuar críticamente atendiendo sus inquietudes y consideraciones controversiales que resultaran de los temas que se van desplegando; apoyando su juicio y exaltando su potencialidad y creatividad al dar alternativas para llegar a la solución a un problema

\section{Metodologia.}

El enfoque en que se orientará la presente investigación es el cualitativo, porque es de carácter social, permite establecer la relación entre el sujeto de estudio y el entorno; a través del análisis e interpretación deductiva se identifica si como resultado de esta interrelación concurre una consecuencia en la población objeto de estudio, orienta al descubrimiento de la hipótesis y es holístico.

- La investigación se ha realizado donde se producen los acontecimientos, para obtener información veraz y oportuna a través de encuestas, entrevistas y observación.

- Además, se concurrió a fuentes escritas con el propósito de detectar, ampliar y profundizar diferentes enfoques, teorías, conceptualizaciones y criterios de varios autores sobre el problema detectado, basándose en documentos, libros, revistas y otras publicaciones. Su desarrollo se basa en consultas bibliográficas, y de campo, y la técnica que se utilizará es el fichaje, mediante fichas mixtas sobre los aspectos del tema.

En la obtención de datos para investigar sobre las variables de estudio y los valores de los indicadores, la técnica que se utilizo es la encuesta y el instrumento, un cuestionario.

Para la investigación de la herramienta para la enseñanza de la geometría utilizando TICS, se eligió muestras mediante el método científico y el diseño cuasi-experimental, a 175 estudiantes y 5 docentes, de una población de estudiantes de segundo año de bachillerato y 
docentes del área de matemáticas, específicamente que dictan la cátedra de geometría en la Unidad Educativa "Pedro Vicente Maldonado", mediante la utilización de la estadística descriptiva, en el que se detallan las alternativas consideradas en las variables de estudio.

Con la información obtenida a través de una encuesta, se analizó los resultados mediante la elaboración de tablas y gráficos estadísticos, que permitieron comprender e interpretar los datos recopilados, donde se analizaron los resultados y se identificaron las preguntas directrices, dando paso a la verificación de las variables mediante la utilización del Chicuadrado, permitiendo validar la hipótesis planteada y contar con elementos básicos para estructurar la propuesta.

\section{Población y muestra}

\section{La población}

Según (Tamayo \& Tamayo. 2004). "La población es la totalidad del fenómeno a estudiar en donde las unidades de población poseen una característica común, la cual se estudia y da origen a los datos de la investigación".

La población de estudio perteneciente a los estudiantes del segundo año bachillerato de la Unidad Educativa "Pedro Vicente Maldonado", asciende a 175 estudiantes.

Para efectos de análisis se determina la muestra de los estudiantes del segundo año bachillerato de la Unidad Educativa "Pedro Vicente Maldonado", y con respecto a los docentes se trabaja con el total de la población para obtener de esta manera datos relevantes.

Tabla 1 Población

\begin{tabular}{ccc}
\hline VARIABLE & FRECUENCIA & PORCENTAJE \\
\hline $\begin{array}{c}\text { Segundo año } \\
\text { Bachillerato A }\end{array}$ & 32 & 18 \\
Segundo año & 36 & 21 \\
Bachillerato B & & \\
Segundo año & 35 & 20 \\
Bachillerato C & & \\
Segundo año & 37 & 21 \\
Bachillerato D & & \\
Segundo año & 35 & 20 \\
Bachillerato E & & $\mathbf{1 0 0 \%}$ \\
TOTAL & $\mathbf{1 7 5}$ & \\
\hline
\end{tabular}

Fuente: Listado de estudiantes matriculados en segundo año bachillerato

\section{Muestra}

Formula 


$$
\mathrm{n}=\frac{z^{2} N(P)(Q)}{\mathrm{E}^{2}(\mathrm{~N}-1)+Z^{2}(\mathrm{P})(\mathrm{Q})}
$$

En donde:

$\mathrm{Z}=$ Margen de Confiabilidad. $(1,65)$

$\mathrm{P}=$ Probabilidad de ocurrencia. $(0,50)$

$\mathrm{Q}=$ Probabilidad de no ocurrencia. $(0,50)$

$\mathrm{E}=$ Error Muestral. $(10 \% ; 0,1)$

$\mathrm{N}$ = Población o universo de estudio. (175)

$(\mathrm{N}-1)=$ Factor de correlación.

Aplicación de la fórmula de la muestra

$\mathrm{Z}=1.65$

$\mathrm{P}=0.50$

$\mathrm{Q}=0.50$

$\mathrm{E}=0.1$

$\mathrm{N}=175$

$(\mathrm{N}-1)=(175-1)$

$\mathrm{n}=\frac{1.65^{2}(175)(0,50)(0,50)}{0,1^{2}(175-1)+1,65^{2}(0,50)(0,50)}$

$n=49,206$

$\boldsymbol{n}=50$ (Encuestados)

Inmediatamente de aplicada la fórmula de la muestra para un universo finito se totaliza una población de 175 unidades de análisis a los cuales realizaremos las encuestas a 50 de ellos

\section{Verificación de hipótesis}

Para la verificación hipotética se utilizó el nivel de $\alpha=0.05$. Tomamos como muestra a 50 estudiantes de segundo año bachillerato de la Unidad Educativa "Pedro Vicente Maldonado" y 5 docentes del área de matemática.

Se procedió a determinar los grados de libertad considerando que el cuadro tiene 5 filas y 3 columnas por lo tanto serán:

$\mathrm{gl}=(\mathrm{f}-1)(\mathrm{c}-1)$

$\mathrm{gl}=(5-1)(3-1)$

$\mathrm{gl}=8$ 
Por lo tanto con 8 grados de libertad y un nivel de 0.05 la tabla del $X^{\wedge} 2 t=15,51$ Entonces; si $X^{\wedge} 2 \mathrm{t} \leq \mathrm{X}^{\wedge} 2 \mathrm{c}$, se aceptará la $\mathrm{H} 0$ caso contrario se la rechazará. $\mathrm{X}^{\wedge} 2 \mathrm{t}=$ 15,51 .

Tabla 2 de Chi cuadrado

\begin{tabular}{|c|c|c|c|}
\hline $\mathbf{D F}$ & $\mathbf{P}=\mathbf{0 , 0 5}$ & $\mathbf{P}=\mathbf{0 , 0 1}$ & $\mathbf{P}=\mathbf{0 , 0 0 1}$ \\
\hline $\mathbf{1}$ & 3,84 & 6,64 & 10,83 \\
\hline $\mathbf{2}$ & 5,99 & 9,21 & 13,82 \\
\hline $\mathbf{3}$ & 7,82 & 11,35 & 16,27 \\
\hline $\mathbf{4}$ & 9,49 & 13,28 & 18,47 \\
\hline $\mathbf{5}$ & 11,07 & 15,09 & 20,52 \\
\hline $\mathbf{6}$ & 12,59 & 16,81 & 22,46 \\
\hline $\mathbf{7}$ & 14,07 & 18,48 & 24,32 \\
\hline $\mathbf{8}$ & 15,51 & 20,09 & 26,13 \\
\hline $\mathbf{9}$ & 16,92 & 21,67 & 27,88 \\
\hline $\mathbf{1 0}$ & 18,31 & 23,21 & 29,59 \\
\hline $\mathbf{1 1}$ & 19,68 & 24,73 & 31,26 \\
\hline
\end{tabular}

Fuente: Tabla de Chi cuadrado

La podemos graficar de la siguiente manera.

Figura 2 Regiones de Chi cuadrado

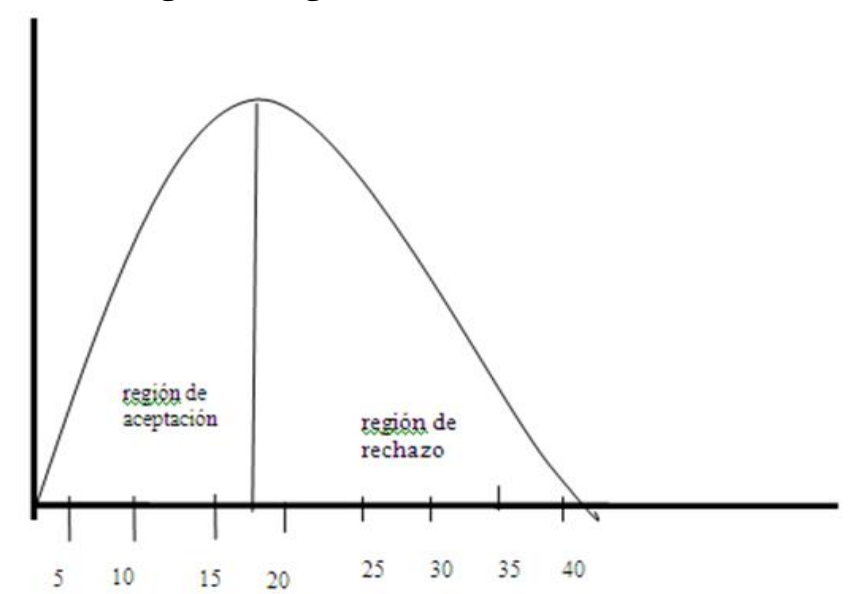

Tabla 3 Cuadro del Chi-Cuadrado estudiantes

\begin{tabular}{ccccc}
\hline $\mathbf{O}$ & $\mathbf{E}$ & $(\mathbf{O}-\mathbf{E})$ & $(\mathbf{O}-\mathbf{E})^{\wedge} \mathbf{2}$ & $(\mathbf{O - E})^{\wedge} \mathbf{2} / \mathbf{E}$ \\
\hline 10 & 18,6 & $-8,6$ & 73,96 & 3,9763 \\
35 & 20 & 15 & 225 & 11,2500 \\
0 & 7,8 & $-7,8$ & 60,84 & 7,8000 \\
10 & 18,6 & $-8,6$ & 73,96 & 3,9763 \\
\hline
\end{tabular}




\begin{tabular}{ccccc}
\hline 35 & 20 & 15 & 225 & 11,2500 \\
0 & 7,8 & $-7,8$ & 60,84 & 7,8000 \\
6 & 18,6 & $-12,6$ & 158,76 & 8,5355 \\
11 & 20 & -9 & 81 & 4,0500 \\
28 & 7,8 & 20,2 & 408,04 & 52,3128 \\
30 & 18,6 & 11,4 & 129,96 & 6,9871 \\
12 & 20 & -8 & 64 & 3,2000 \\
3 & 7,8 & $-4,8$ & 23,04 & 2,9538 \\
37 & 18,6 & 18,4 & 338,56 & 18,2022 \\
7 & 20 & -13 & 169 & 8,4500 \\
1 & 7,8 & $-6,8$ & 46,24 & 5,9282 \\
$\mathbf{2 2 5}$ & $\mathbf{2 3 2}$ & & & $\mathbf{1 5 6 , 6 7 2 3}$ \\
\hline
\end{tabular}

Tabla 4 Frecuencias observadas

\begin{tabular}{ccccc}
\hline $\mathbf{O}$ & $\mathbf{E}$ & $\mathbf{( O - E )}$ & $(\mathbf{O}-\mathbf{E})^{\wedge} \mathbf{2}$ & $(\mathbf{O - E})^{\wedge} \mathbf{2} / \mathbf{E}$ \\
\hline 0 & 2 & -2 & 4 & 2,0000 \\
3 & 1,4 & 1,6 & 2,56 & 1,8286 \\
1 & 0,6 & 0,4 & 0,16 & 0,2667 \\
1 & 2 & -1 & 1 & 0,5000 \\
3 & 1,4 & 1,6 & 2,56 & 1,8286 \\
0 & 0,6 & $-0,6$ & 0,36 & 0,6000 \\
4 & 2 & 2 & 4 & 2,0000 \\
0 & 1,4 & $-1,4$ & 1,96 & 1,4000 \\
0 & 0,6 & $-0,6$ & 0,36 & 0,6000 \\
4 & 2 & 2 & 4 & 2,0000 \\
0 & 1,4 & $-1,4$ & 1,96 & 1,4000 \\
0 & 0,6 & $-0,6$ & 0,36 & 0,6000 \\
1 & 2 & -1 & 1 & 0,5000 \\
1 & 1,4 & $-0,4$ & 0,16 & 0,1143 \\
2 & 0,6 & 1,4 & 1,96 & 3,2667 \\
\hline
\end{tabular}

Fuente: Frecuencias observadas

Con $8 \mathrm{gl}$ con un nivel de $0,05 \quad \mathrm{X}^{\wedge} 2 \mathrm{t}=15,51$

$\mathrm{X}^{\wedge} 2 \mathrm{c}=156,67$ en el caso de los estudiantes y 18,90 en el caso de los docentes de acuerdo a las regiones planteadas los últimos valores son mayores que el primero y se hallan por lo tanto en la región de rechazo, se rechaza la hipótesis nula y se acepta la hipótesis alterna que dice:

"H1: La guía didáctica como herramienta en el proceso de enseñanza de la geometría utilizando TICS si mejora positivamente en el rendimiento académico, de los estudiantes de segundo año bachillerato de la Unidad Educativa "Pedro Vicente Maldonado." 


\section{Resultados}

Encuesta aplicada a estudiantes

¿Cree usted que el uso de TICS mejoraría el aprendizaje de la geometría?

Tabla 5 Encuesta

\begin{tabular}{lll}
\hline ALTERNATIVAS & FRECUENCIA & PORCENTAJE \\
\hline Siempre & 36 & 72 \\
A Veces & 14 & 28 \\
Nunca & 0 & 0 \\
TOTAL & 50 & $100 \%$ \\
\hline
\end{tabular}

Fuente: Encuesta

Del número de encuestados el $72 \%$ considera que siempre el uso de las TICS mejoraría el aprendizaje de la geometría y el $28 \%$ en cambio opina que a veces.

Lo cual nos da una clara muestra que la incorporación y el uso de las TICS en las aulas, son una herramienta interesante e innovadora que mejoraría el aprendizaje de la geometría.

Tabla 6 Considera necesaria la utilización de TICS

\begin{tabular}{lll}
\hline ALTERNATIVAS & FRECUENCIA & PORCENTAJE \\
\hline Siempre & 35 & 70 \\
A Veces & 15 & 30 \\
Nunca & 0 & 0 \\
TOTAL & 50 & $100 \%$ \\
\hline
\end{tabular}

Fuente: Encuesta

El 70\% de estudiantes encuestados opinan que siempre es necesaria la utilización de TICS en la enseñanza aprendizaje de la geometría, el 30\% indican a veces, el $0 \%$ dice que nunca. Los estudiantes sienten la necesidad de trabajar con las TICS en geometría, debido al acceso y dominio que los estudiantes tienen sobre los recursos tecnológicos, lo cual sería un punto a su favor y facilitaría la revisión de contenidos de esta asignatura.

Tabla 7 Encuesta

\begin{tabular}{lll}
\hline ALTERNATIVAS & FRECUENCIA & PORCENTAJE \\
\hline Siempre & 6 & 12 \\
A Veces & 17 & 34 \\
Nunca & 27 & 54 \\
TOTAL & 50 & $100 \%$ \\
\hline
\end{tabular}

Fuente: Encuesta 
El 54\% de estudiantes encuestados indican que nunca utilizan las TICS su maestro para el tratamiento de su asignatura, el $34 \%$ opinan que a veces y, el 12\% indica que siempre.

Las TIC, son una herramienta valiosa de trabajo, que los docentes debemos estar capacitados para poder acceder a los mismos e impartir nuestros conocimientos con herramientas que sean interesantes y atractivas para los estudiantes, pues es a ellos a quienes dirigimos el conocimiento.

\section{Conclusiones.}

- De acuerdo a la investigación realizada, se da a conocer conclusiones a las que he llegado a determinar:

- El uso de las tecnologías de la información y estrategias tecnológicas innovadoras de amplia cobertura permitirán el enriquecimiento profesional en los educadores, fomentando la reflexión sobre los programas del plan Estudio vigente en la asignatura de geometría que es una rama de la matemática que se ocupa del estudio de las propiedades de las figuras en el plano o el espacio, incluyendo: puntos, rectas, planos, politopos (que incluyen paralelas, perpendiculares, curvas, superficies, polígonos, poliedros, etc.).

- La capacitación del docente en el área de matemática es imprescindible para fortalecer el aprendizaje en los educandos, contribuyendo en la mejora de la educación, es así que el $72 \%$ de estudiantes y el $80 \%$ de docentes encuestados, consideran que el uso las TICS, mejoraría el aprendizaje de la geometría y su rendimiento.

- El empleo de un software educativo en las actividades matemáticas se ha generalizado de manera notable debido a los avances en su desarrollo, y aplicaciones informáticas creadas con la finalidad de ser utilizados como medio didáctico, para facilitar los procesos de enseñanza y de aprendizaje, determinándose esto en la presente investigación lo cual resalta que el $100 \%$ de docentes en su encuesta considera que es necesario desarrollar una guía didáctica con la aplicación del TICS para determinar el óptimo aprendizaje de la geometría y poder comprobar los cambios en el estudiante. Los educandos investigados se muestran motivados por participar activamente en la construcción de los procesos de enseñanza aprendizaje a través de la aplicación de propuestas metodológicas innovadoras.

\section{Referencias bibliográficas.}

- Apostol, T. M. (1973); Calculos Vol 1 y Vol 2. Barcelona. Reverté.

- Benalcazar, H. (2007); Matemática para el Bachillerato. Quito.

- Bosch, G. C. (1998); Matemáticas básicas. SEP Coanlep. Limusa. México.

- Dennis Zill (2000); Cálculo con Geometría Analítica; México: Iberoamericana

- Dinacaped, (1999); Didáctica de la Matemática, Quito.

- Drooyan, I; Franklin, K. (1998); Elementos de álgebra para bachillerato. Limusa. México.

- Feldman, R. (2005) "Psicología: con aplicaciones en países de habla hispana". (Sexta 
- Edición) México, McGrawHill.

- Galindo, E. (1999); Estadística para la Administración y la Ingeniería. Quito.

- Gustafson, D. R. (1997); Álgebra intermedia. Thomson. México.

- Gutiérrez, J.L. (1998); Matemática básica, moderna y geometría. España.

- Lara, A. Arroba, J. (2010); Análisis matemático. Centro de Matemática de la Universidad Central del Ecuador

- Lehmann, H. C. (2001); Álgebra. Limusa. México.

- Lovaglia, F. (1972); Álgebra. Harla. México.

- Pérez Seguí, M. L. (2003); Teoría de números. Cuadernos de olimpiadas de matemáticas. Instituto de matemáticas. Universidad Nacional Autónoma de México.

- Sadler, A. J.; Thorning, D.W.S. (1994); Understing pure mathematics. Oxford University Press. UK

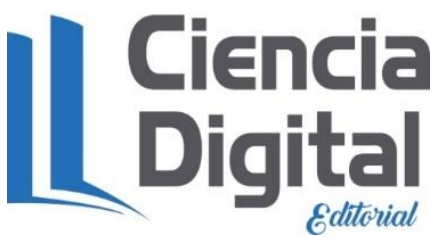




\section{Para citar el artículo indexado.}

Cachuput Gusñay, J., Martínez Nogales, J., Guilcapi Mosquera, J., \& López Ortega, J. (2019). Herramienta para la enseñanza de la geometría utilizando Tics, dirigido a los estudiantes del segundo año de bachillerato. Explorador Digital, 3(3.1), 41-58. https://doi.org/10.33262/exploradordigital.v3i3.1.864

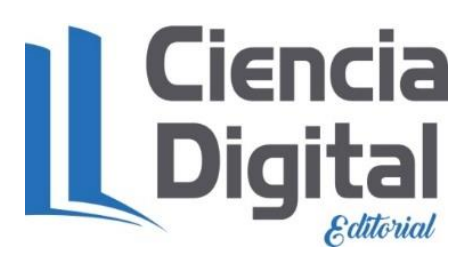

El artículo que se publica es de exclusiva responsabilidad de los autores y no necesariamente reflejan el pensamiento de la Revista Explorador Digital.

El articulo queda en propiedad de la revista y, por tanto, su publicación parcial y/o total en otro medio tiene que ser autorizado por el director o editor de la Revista

\section{Explorador Digital.}
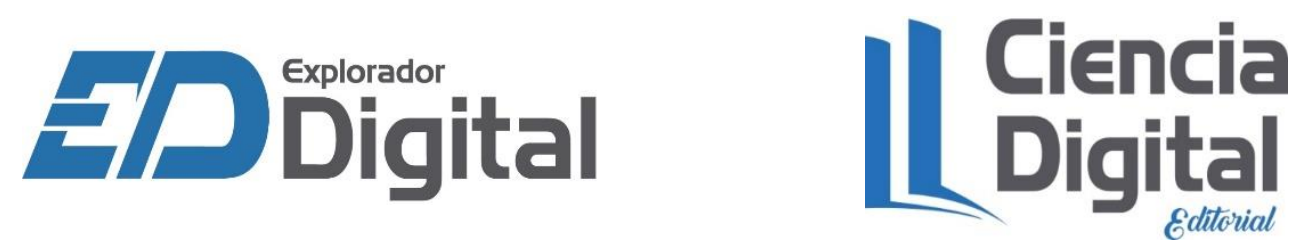